14. Pennetti V, Paolucci AM, Spadoni MA. Patterns and nutritional implications of the free serum amino acids in a pygmy adult population of Bagandu Central African Republic pygmies. In: Cavalli-Sforza LL, ed. African pygmies. Orlando, Fla.: Academic Press, 1986:143-52.

15. Merimee TJ, Zapf J, Hewlett B, Cavalli-Sforza LL. Insulin-like growth factors in pygmies: the role of puberty in determining final stature. $N$ Engl $J$ Med 1987; 316:906-11.

16. Baumann G, Shaw MA, Ambum K. Regulation of plasma growth hormonebinding proteins in health and disease. Metabolism (in press)

17. Zapf J, Walter H, Froesch ER. Radioimmunological determination of insulinlike growth factor I and II in normal subjects and in patients with growth disorders and extrapancreatic tumor hypoglycemia. J Clin Invest 1981; 68:1321-30.

18. Baumann G, Shaw MA, Buchanan TA. In vivo kinetics of a covalent growth hormone-binding protein complex. Metabolism $1989 ; 38: 330-3$

19. Tanner JM, Whitehouse RH, Takaishi M. Standards from birth to maturity for height, weight, height velocity, and weight velocity: British children, 1965. Arch Dis Child 1966; 41:454-71, 613-35.

20. Kahn CR. Insulin resistance, insulin insensitivity, and insulin unresponsive ness: a necessary distinction. Metabolism 1978; 27:Suppl 2:1893-902.

21. Kolterman OG, Insel J, Saekow M, Olefsky JM. Mechanisms of insulin resistance in human obesity: evidence for receptor and postreceptor defects. J Clin Invest 1980; 65:1272-84.

?2. Crettaz M, Jeanrenaud B. Postreceptor alterations in the states of insulin resistance. Metabolism 1980; 29:467-73.

23. Bierich JR, Moeller H, Ranke MB, Rosenfeld PG. Pseudopituitary dwarfism due to resistance to somatomedin: a new syndrome. Eur J Pediatr 1984, 142:186-8.

24. Mann GV. The riddle of pygmy stature. N Engl J Med 1987; 317:709-10.
25. Finkelstein JW, Roffwarg HP. Boyar RM, Kream J, Hellman L. Agerelated change in the twenty-four-hour spontancous secretion of growth hormone. J Clin Endocrinol Metab 1972; 35:665-70.

26. Mauras N, Blizzard RM, Link K, Johnson ML, Rogol AD, Veldhuis JD. Augmentation of growth hormone secretion during puberty: evidence for a pulse amplitude-modulated phenomenon. J Clin Endocrinol Metab 1987; 64:596-601.

27. Luna AM, Wilson DM, Wibbelsman CJ, et al. Somatomedins in adoles cence: a cross-sectional study of the effect of puberty on plasma insulinlike growth factor I and II levels. J Clin Endocrinol Metab 1983; 57:26871.

28. Miller JD, Tannenbaum GS, Colle E, Guyda HJ. Daytime pulsatile growth hormone secretion during childhood and adolescence. J Clin Endocrinol Melab 1982. 55:989-94.

29. Underwood LE, Van Wyk JJ. Normal and aberrant growth. In: Wilson JD, Foster DW, eds. Williams textbook of endocrinology. 7th ed. Philadelphia: W.B. Saunders, 1985:167.

30. Parker MW, Johanson AJ, Rogol AD, Kaiser DL, Blizzard RM. Effect of testosterone on somatomedin-C concentrations in prepubertal boys. J Clin Endocrinol Metab 1984; 58:87.90.

31. Clemmons DR, Klibanski A, Underwood LE, et al. Reduction of plasma immunoreactive somatomedin $\mathrm{C}$ during fasting in humans. J Clin Endocrinol Metab 1981; 53:1247-50.

32. Baumann G, Shaw MA. Merimee TJ, Clemmons DR. Growth hormone binding protein in human plasma: downregulation by prolonged fasting in lean but not obese subjects. Clin Res 1988; 36:477 A. abstract.

33. Baumann G, Amburn KD, Buchanan TA. The effect of circulating growth hormone-binding protein on metabolic clearance, distribution and degradation of human growth hormone. J Clin Endocrinol Metab 1987; 64:657-60.

\title{
EFFICACY OF LIVER TRANSPLANTATION IN PATIENTS WITH PRIMARY BILIARY CIRRHOSIS
}

\author{
Bernd H. Markus, M.D., E. Rolland Dickson, M.D., Patricia M. Grambsch, Ph.D., \\ Thomas R. Fleming, Ph.D., Vincenzo Mazzaferro, M.D., Goran Bo G. Klintmal.h, M.D., \\ Russell H. Wiesner, M.D., David H. Van Thiel, M.D., \\ and Thomas E. Starzl, M.D., Ph.D.
}

\begin{abstract}
iusick
icians

: Afri-

olated

5:965-

pecific

zation.

tion of

$J$ Clin

i peak

nology

(GH)-

Endo-

tor and

: 1987;

plasma

recep-

binding

dwarf-

th hor-

Metab

model. All patients had undergone liver transplantation between March 1980 and June 1987 and were

TVER transplantation has been accepted clinically 1 as a lifesaving treatment in various end-stage liver diseases, including primary biliary cirrhosis. ${ }^{1,2}$ However, no controlled trials have been performed to

From the Department of Surgery (B.H.M., V.M., T.E.S.), the Division of Gastroenterology, and the Department of Internal Medicine (D.H.V.T.), University of Pittsburgh School of Medicine, Pittsburgh; the Division of Gastroenterology and Internal Medicine (E.R.D., R.H.W.) and the Section of Biostatistics (P.M.G.), Mayo Clinic and Mayo Foundation, Rochester, Minn.; the Department of Biostatistics, University of Washington, Seattle (T.R.F.); and the Department of Surgery, Baylor University Medical Center, Dallas (G.B.G.K.). Address reprint requests to Dr. Starzl at the Department of Surgery, University of Pittsburgh School of Medicine, Falk Clinic, 3601 Fifth Ave., Pittsburgh, PA 15213

Supported by a research grant (DK-342-38-02) from the National Institutes of Health. Dr. Markus has a research fellowship from the Deutsche Forschungsgemeinschaft.
\end{abstract}

followed for a median of 25 months.

Three months after liver transplantation, the KaplanMeier survival probabilities in the recipients were substantially higher than the Mayo-model "simulated-control" survival probabilities $(P<0.001)$. At two years, the Kaplan-Meier survival probability was 0.74 , whereas the mean Mayo-model survival probability was 0.31 . The patients who were at low risk according to the Mayo model had the best probability of survival after liver transplantation; however, patients at all risk levels who had undergone liver transplantation had higher probabilities of survival than those who had not. We conclude that liver transplantation is an efficacious treatment in patients with advanced primary biliary cirrhosis. (N Engl J Med 1989; 320:1709-13.) evaluate the efficacy of this procedure. Indeed, because there has been a marked improvement since 1981 in survival after transplantation, random assignment of patients with advanced liver disease to a nontransplantation control group is considered to be clinically inappropriate.

At the Mayo Clinic, a Cox regression model for predicting the probability of survival in patients with conservatively treated primary biliary cirrhosis has been developed. ${ }^{3}$ To provide control data for assessing the efficacy of liver transplantation in improving survival, this model was applied to the combined population of patients with primary biliary cirrhosis who underwent transplantation at the University of 
Table 1. Values for Mayo-Model Variables in 161 Patients with Primary Biliary Cirrhosis Classified in Risk Subgroups.

\begin{tabular}{lcccc}
\hline \hline VARIABLB & $\begin{array}{c}\text { Group } 1 \\
(\mathrm{~N}=98)\end{array}$ & $\begin{array}{c}\text { Group } 2 \\
(\mathrm{~N}=41)\end{array}$ & $\begin{array}{c}\text { Group } 3 \\
(\mathrm{~N}=22)\end{array}$ & $\begin{array}{c}\text { ALL Groups } \\
(\mathrm{N}=161)\end{array}$ \\
& & \multicolumn{2}{c}{ mean $\pm S D$} \\
Age (yr) & $46.5 \pm 8.5$ & $47.8 \pm 6.8$ & $53.8 \pm 9.0$ & $47.8 \pm 8.5$ \\
Bilirubin (mg/dl)* & $12.1 \pm 8.2$ & $24.1 \pm 12.2$ & $27.8 \pm 11.7$ & $17.3 \pm 11.8$ \\
Albumin (g/d) & $3.1 \pm 0.5$ & $2.7 \pm 0.6$ & $2.5 \pm 0.4$ & $2.9 \pm 0.6$ \\
Prothrombin time (sec) & $13.4 \pm 1.4$ & $15.1 \pm 2.5$ & $19.5 \pm 5.7$ & $14.6 \pm 3.4$ \\
Edema score $\dagger$ & $0.4 \pm 0.4$ & $0.8 \pm 0.4$ & $0.9 \pm 0.2$ & $0.5 \pm 0.4$ \\
Risk score $\dagger$ & $7.4 \pm 1.0$ & $9.2 \pm 0.4$ & $10.4 \pm 0.5$ & $8.2 \pm 1.4$ \\
\hline
\end{tabular}

-To convert to micromoles per liter, multiply by $\mathbf{1 7 . 1}$.

tAccording to the Mayo model.

vival curve for the 161 patients who had primary biliary cirrhosis and the averaged Mayo-model curve for predicted survival. The two curves were similar during the first three months after transplantation, a period during which the actual post-transplantation survival probability decreased sharply. The curves diverged later in the post-transplantation course. At one year after transplantation, the mean $( \pm S D)$ actual survival probability was $0.76 \pm 0.03$, and the averaged Mayo-model survival probability was 0.45 . At two years these values were $0.74 \pm 0.04$ and 0.31 , respectively. The one-sample log-rank test showed the difference between actual survival and Mayo-model survival to have a high level of significance $\left(\chi^{2}=372.5\right.$, $\mathrm{P}<0.001$ ).

In Figure 2, actual survival curves are compared with average survival curves based on the Mayo model for each of the three risk groups. At each risk level, the actual survival beyond three months after transplantation was better than the survival predicted by the Mayo model $(\mathrm{P}<0.0001$ in all cases).

Figure 2 shows clearly that patients who had lower risk scores according to the Mayo model (i.e., those who had better prognoses according to the model for survival with no treatment) also had better post-transplantation survival. A Cox regression analysis assess-

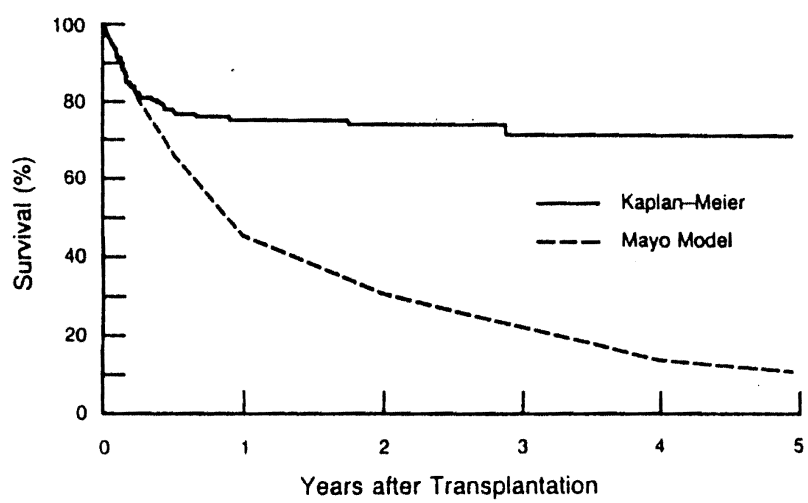

Figure 1. Actual (Kaplan-Meier) Survival after Transplantation in 161 Patients with Primary Biliary Cirrhosis and Estimated Survival without Transplantation as Predicted by the Mayo Model (Simulated Control). ing the association of the risk score with actual posttransplantation survival indicated that each unit increase in the risk score increased the death rate by a factor of $e^{0.27}=1.30$ (95 percent confidence interval, 1.04 to 1.65). When the analysis was restricted to the first 90 days after transplantation, the factor increased to 1.63 (95 percent confidence interval, 1.23 to 2.15 ), showing the greater influence of risk on short-term survival.

Twenty-eight of the 29 patients who underwent transplantation for reasons other than poor liver function were in Group 1, the group with the lowest risk. When the patients in Group 1 were subclassified according to the primary indication for liver transplantation (poor liver function or associated complications), those who had poor liver function were found to be at higher risk in terms of the risk factors - notably

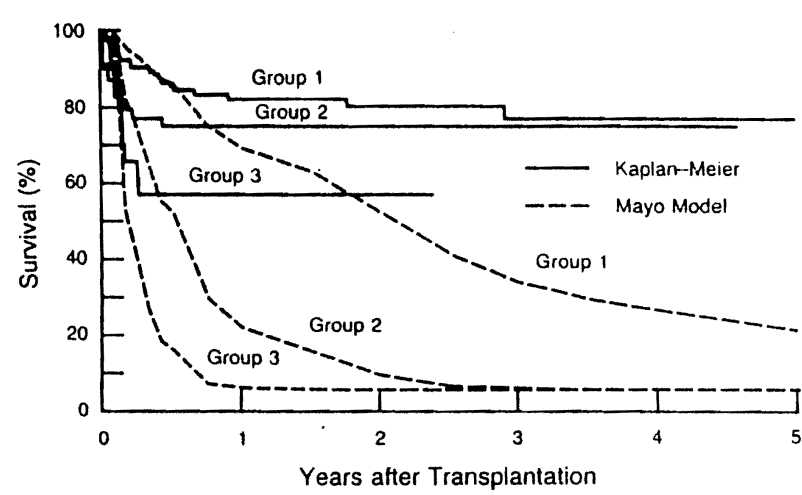

Figure 2. Actual (Kaplan-Meier) Survival after Transplantation in Three Risk Groups of Patients with Primary Biliary Cirrhosis and Estimated Survival without Transplantation as Predicted by the Mayo Model.

The risk groups were formed on the basis of pretransplantation Mayo-model risk scores. Group 1 (low risk) comprised 98 patients with risk scores below 8.67; Group 2 (medium risk), 41 patients with scores between 8.67 and 9.93; and Group 3 (high risk), 22 patients with scores above 9.93 .

the bilirubin level (Table 2). The patients who underwent transplantation because of poor liver function had a significantly higher survival rate than that predicted by the Mayo model ( $\mathrm{P}<0.0001$ by one-sample log-rank test) (Fig. 3). However, survival after transplantations performed for other reasons was similar to that predicted by the Mayo model ( $\mathrm{P}=0.376$ by onesample log-rank test).

There was evidence of an improvement in outcome over time. The group of 122 patients who underwent transplantation after the introduction of OKT3 therapy in November $1984^{13}$ had better survival (oneyear Kaplan-Meier probability, 0.79 \pm 0.04 ) than the 39 patients who underwent transplantation before that time $(0.64 \pm 0.08)$. The difference in survival between these two periods remained statistically significant after adjustment for the risk score. The adjusted death rate in the later period was estimated as 53 
Pittsburgh and at the Baylor University Hospital in Dallas. Actual survival after transplantation was compared with the survival estimated with use of the Mayo model.

\section{Methods}

\section{Mayo Model}

The control group used in our evaluation of the effect of liver transplantation on survival was formed according to data generated by the Mayo model for assessing survival in relation to the natural history of primary biliary cirrhosis. ${ }^{3}$ This is a Cox regression model ${ }^{4,5}$ that uses a small number of inexpensive, noninvasive, and universally available measurements to predict survival in patients in whom this disease has not been treated. It was developed from a data base on 312 patients with primary biliary cirrhosis who had been referred to the Mayo Clinic between January 1974 and May 1984 and who met standard eligibility criteria for a randomized controlled clinical trial comparing $D$-penicillamine with a placebo. Because this drug is now considered to be ineffective in primary biliary cirrhosis, ${ }^{6}$ having no apparent effect on survival, biochemical changes, or histologic progression, all 312 patients were considered to have received standard care (which has at most a negligible effect on the prognosis). Of these patients, 10 percent had undergone sclerotherapy or shunt procedures to control severe bleeding; these interventions are not thought to alter survival meaningfully. Only 6 percent of the 312 patients received a liver transplant, and the follow-up of these patients was censored at transplantation.

The model was cross-validated in an independent patient population - 106 patients who were eligible for the clinical trials but who chose not to participate. The model fit the data on survival well. As a result, the model was enhanced by combining the patients in the trial and outside it into a large group of 418 subjects for an estimation of the values of model variables. The model was also found to assess risk in a way similar to that of other models that used Cox regression to evaluate survival in primary biliary cirrhosis. ${ }^{7,8}$ Finally, extramural cross-validation in a group of 176 patients with the disease (141 from the New England Medical Center hospitals [Boston] and 35 from the Scott and White Clinic [Temple, Tex.]) established the generalizability of the model (unpublished data).

The Mayo model uses the patient's age and four measures of liver function - total serum bilirubin and serum albumin concentrations, prothrombin time, and a score for the clinical severity of edema. It combines these five variables to obtain a risk score $(R)$ for each patient, in the following equation: $R=0.871 \log _{\text {c }}$ (bilirubin in milligrams per deciliter) $-2.53 \log _{\mathrm{e}}$ (albumin in grams per deciliter) +0.039 (age in years) $+2.38 \log _{c}$ (prothrombin time in seconds) +0.859 (edema score).

The edema score is determined as follows: 0 , minimal edema, defined as either edema requiring no diuretic therapy or as absence of edema; 0.5 , moderate edema, defined as edema that resolved after treatment with diuretic agents, or edema for which no diuretic therapy was prescribed; and I, severe edema, defined as edema that persisted despite treatment with diuretic agents.

The risk score is then used to produce a predicted-survival probability curve for that risk level. This curve indicates the probability that a patient will survive a given number of years after the risk score has been measured. Technical details are available elsewhere. $^{3}$

\section{Patient Population}

Between March 1980 and June 1987, 981 patients underwent primary liver transplantation at the University of Pittsburgh or Baylor University, as described by Starzl et al. ${ }^{9}$ All the patients received base-line immunosuppressive treatment with cyclosporine and steroids. Beginning in November 1984, OKT3 monoclonal antibody was used to treat severe episodes of transplant rejection.

Before transplantation, a diagnosis was established in each patient on the basis of clinical evaluation, biochemical values, histologic assessment, and additional testing. Of the 981 patients, 161 were given a diagnosis of primary biliary cirrhosis ( 149 women $[93$ percent] and 12 men [ 7 percent]; all the patients except four were white [three black women and one black man]). These 161 patients were treated between April 1980 and June 1987; the median month of transplantation was November 1985. The five variables for the Mayo model were selected from the data in the patients' medical records for the period immediately preceding transplantation. Follow-up was completed in these patients as of December 18, 1987. The median duration of follow-up after transplantation was 25 months.

In 132 of the 161 patients with primary biliary cirrhosis, the primary indication for transplantation was poor liver function However, in the remaining 29 patients, additional complications had led to transplantation: major esophageal varices with repeated massive bleeding of the upper gastrointestinal tract, necessitating blood transfusions, despite repeated sclerotherapy ( 25 patients), and advanced osteodystrophy (4 patients)

\section{Statistical Analysis}

Survival time was defined as the time that elapsed from the initial liver transplantation until death or the date on which follow-up ended, whichever occurred first. All patients were included in the analysis, including those who received multiple transplants; the lat ter were given no special treatment. Thirty-three patients had undergone transplantation twice, and 7 others three times

Actual post-transplantation survival was estimated with the Kaplan-Meier product-limit estimator. ${ }^{10}$ Predicted survival without transplantation was computed for each patient with use of the Mayo model. The values for actual and model-predicted survival were compared in two ways - graphically and by means of a statistical test.

For the graphic method, the individual probabilities for predicted survival were averaged to produce a mean Mayo-model survival curve, to be compared with the Kaplan-.. feier curve. For purposes of presentation. the averaged Mavo-model survival probabilities were computed at only a few time points after transplantation three months, six months, and at yearly intervals - and then interpolated linearly

Statistical testing of differences between the survival predicted with the Mayo model and the actual survival was performed with the one-sample log-rank test," as implemented in the S.AS Institute's supplemental procedure SURVDIFF. ${ }^{12}$ This test uses the Mayo-model predicted-survival curve for each patient as a mathematical control for that patient.

For further analysis, the 161 recipients of liver transplants were classified into three risk groups according to Mayo-model risk score. The risk-score cutoff values dividing the groups (8.67 and 9.93) were chosen so that the three groups had roughly equal numbers of deaths that occurred in the first three months after transplantation. Group 1, with the lowest risk, consisted of 98 patients, with 10 deaths in the first three months. Group 2 had 41 patients, with 10 deaths in the first three months. Group 3 had 22 patients, with nine deaths in the first three months.

The relation between the Mayo-model risk score and the actual post-transplantation survival was assessed by two Cox regression analyses. One analysis examined the entire follow-up period, and the other was restricted to the first 90 days after trantsplantation.

Temporal trends in post-transplantation outcome were assessed by subclassifying the patients into the 40 who underwent transplantation before OKT3 was used to treat severe rejection (November 1984) and the 121 who underwent transplantation after that date. Cox regression was used to compare survival in these (ivo groups; differences in risk at base line were controlled for by including the risk score from the Mayo model as a covariate.

\section{RESUlTS}

Table 1 shows the descriptive statistics for the entire study population and for each of the three risk groups. There were 41 deaths overall.

Figure 1 shows the actual post-transplantation sur- 